\title{
Low energy events in NaI(TI) scintillators. ANAIS status and prospects.
}

\author{
J. Amaré, S. Borjabad, S. Cebrián, C. Cuestaํ, D. Fortuño, E. García, C. Ginestra, \\ H. Gómez, M. Martínez, M. A. Oliván, Y. Ortigoza, A. Ortiz de Solórzano, C. Pobes,
}

J. Puimedón, M. L. Sarsa and J. A. Villar.

Grupo de Física Nuclear y Astropartículas. Universidad de Zaragoza. C/ Pedro Cerbuna 12, 50009, Zaragoza, Spain.

E-mail: ccuesta@unizar.es

The search of the annual modulation in the detection rates that could evidence the presence of galactic dark matter energy depositions in the detector is of utmost interest after DAMA/LIBRA positive result [1] and recent hints from other experiments like CDMS [2] and CoGeNT [3]. Another experiment using the same target that DAMA/LIBRA is required in order to confirm the effect: ANAIS is a project to be carried out at the Canfranc Underground Laboratory with such a goal. The complete experiment proposed will use about $250 \mathrm{~kg}$ of $\mathrm{NaI}(\mathrm{Tl})$ crystals to study the expected annual modulation in the galactic dark matter signal. Very high radiopurity levels are required; particularly, very low potassium content in the crystals. For this goal new powder purification techniques are being developed. In parallel, a $9.6 \mathrm{~kg} \mathrm{NaI(Tl)}$ prototype, called ANAIS-0, has been tested underground. With ANAIS-0, low energy events coming from external gamma calibration sources and from the $3.2 \mathrm{keV}$ line of the ${ }^{40} \mathrm{~K}$ internal contamination are studied to develop different low energy estimators and parameters for noise rejection. Tests of different photomultipliers with or without light guides are ongoing in order to determine the best compromise between high light collection and low background.

Identification of Dark Matter 2010 (IDM2010)

Montpellier, France

July 26-30, 2010

\footnotetext{
$1 \quad$ Speaker
} 


\section{ANAIS experiment}

ANAIS is a project aiming to set up, at the new facilities of the Canfranc Underground Laboratory (Spain), a large scale $\mathrm{NaI}(\mathrm{Tl})$ experiment to explore the WIMPs parameter space compatible with the DAMA/LIBRA annual modulation positive result. For this goal, some of the experimental features of ANAIS should be: energy threshold below 2 keVee in order to improve sensitivity for the annually modulated WIMP signal, background at low energy kept as low as possible and very stable operation conditions $[4,5]$. The original proposal consisted of $100 \mathrm{~kg} \mathrm{NaI(Tl)}$ crystals for which funding is allowed but an enlargement up to $250 \mathrm{~kg}$ has been recently proposed.

\subsection{NaI(Tl) powder purification}

Characterization of existing 14 hexagonal crystals (10.7 kg each) stored underground since 1988 has shown that ${ }^{40} \mathrm{~K}$ internal contamination (15 to $20 \mathrm{mBq} / \mathrm{kg}$ ) is too high to use these detectors. New crystals with very low content in potassium should be used in ANAIS requiring the development of new powder purification techniques. In this direction, ANAIS collaboration signed an agreement with Electrochemical Systems Inc. of Knoxville (U.S.A.), to purify commercial NaI powder to less than $0.1 \mathrm{ppm}$ of potassium. This agreement is divided into three phases: Phase I was the determination of possible NaI purification methods. The on-going Phase II is the test of candidate processes and production of reference sample(s); several raw samples of the starting material have been measured at the University of Zaragoza with two methods (HP Ge Spectrometry and Atomic Absorption Spectroscopy). Purified samples will also be checked and analyzed with the same methods. Finally, Phase III will consist on the production and delivery of the total amount of the required product (100-250 kg).

\subsection{ANAIS-0 module}

In a parallel way, a $\mathrm{NaI}(\mathrm{Tl})$ ultrapure crystal $(9.6 \mathrm{~kg})$ made by Saint-Gobain has been encapsulated in the University of Zaragoza as a detector. It has been coupled to two photomultipliers and installed at the Canfranc Underground Laboratory. Measurements are carried out to characterize and fully understand ANAIS background at low energy, optimize noise cuts, determine the calibration method, test the electronics and improve the light collection efficiency. Different photomultipliers, with and without light guides, have been tested with this module. In the following some of the results obtained with such a module will be presented.

\section{Low energy events}

As the expected dark matter signal in $\mathrm{NaI}$ is below 6 keVee, low energy response of the detectors is crucial, requiring a good energy calibration and a powerful noise rejection technique. For this purpose, populations of scintillation events, with known low energy, are very useful. The populations studied come from external gamma calibration sources and from the 
$3.2 \mathrm{keV}$ line of the ${ }^{40} \mathrm{~K}$ internal contamination. The external gamma calibration sources used are: ${ }^{55} \mathrm{Fe}(6.0 \mathrm{keV}),{ }^{109} \mathrm{Cd}(22.1 \mathrm{keV}$ and $88.0 \mathrm{keV}),{ }^{137} \mathrm{Cs}(32.1 \mathrm{keV}),{ }^{57} \mathrm{Co}(6.4 \mathrm{keV}, 14.4 \mathrm{keV}$ and $122.1 \mathrm{keV})$ and ${ }^{133} \mathrm{Ba}(30.9 \mathrm{keV}$ and $81.0 \mathrm{keV})$, see Figure 1a. A Mylar window at ANAIS-0 copper encapsulation allows such low energy calibrations. It is worth noting that below $10 \mathrm{keV}$, surface effects are observed, when using external gamma sources, since the energy measured is lower than the energy from the external source. On the other hand, in measurements carried out in coincidence with one of the $10.7 \mathrm{~kg} \mathrm{NaI}(\mathrm{Tl})$ detectors, ${ }^{40} \mathrm{~K}$ internal contamination is estimated by the coincidence among X-rays and Auger-e (3.2 keV) in the ultrapure crystal $(9.6 \mathrm{~kg}$ ) and $1461 \mathrm{keV}$ in the other one. Selected events provide a very low energy population that can be very useful to study several features of ANAIS-0. For instance, the trigger efficiency achieved can be estimated (see Figure $1 \mathrm{~b}$ ). Also the ${ }^{40} \mathrm{~K}$ bulk activity of the $\mathrm{NaI}(\mathrm{Tl})$ ultrapure crystal has been derived from that measurement to be $12.7 \pm 0.5 \mathrm{mBq} / \mathrm{kg}$.

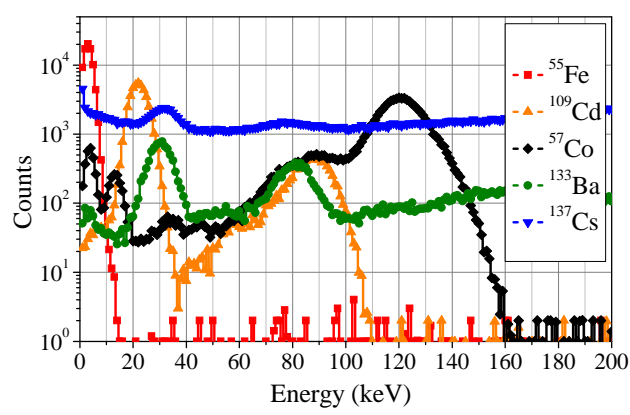

(a)

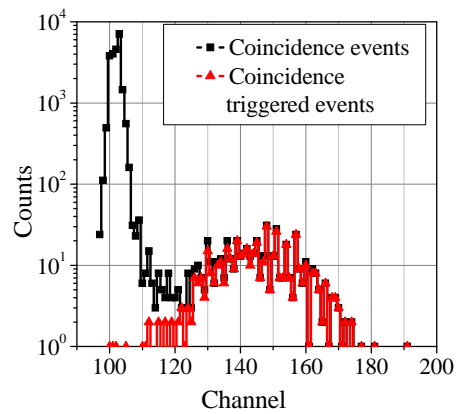

(b)

Figure 1: (a) Spectra of external calibration sources used and (b) low energy spectrum obtained with the coincidence method: events that produced trigger also in the ultrapure crystal (3.2 keV signal) are in red, confirming very high trigger efficiency at this energy.

Different low energy estimators have been studied with the $3.2 \mathrm{keV}$ events: charge converted signal (QDC), number of individual peaks in the digitized pulse (n) and the total area of the peaks in the pulse (area) see Figure 2. QDC signal is the most commonly used, but a combination of some of them could help to improve noise rejection once efficiency is appropriately established.
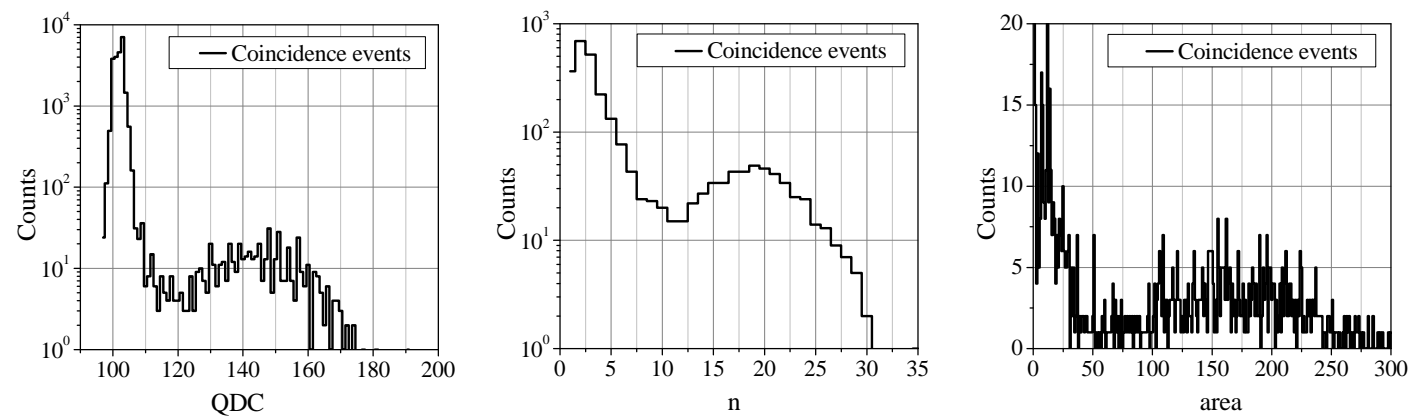

Figure 2: Different low energy estimators applied to the ${ }^{40} \mathrm{~K}$ low energy events. 
In order to be sensitive in the region below 6 keVee, strong rejection of PMT noise events should be guaranteed. Bulk scintillation events have been studied in terms of the timing properties of the pulses using a ${ }^{57} \mathrm{Co}$ source. One of the pulse parameters studied is P1, which measures the ratio of the pulse area in a region from 100 to $600 \mathrm{~ns}$ and from 0 to $600 \mathrm{~ns}$ from the pulse onset. In the plot P1 versus QDC (see Figure 3) the $1 \sigma$ region about the mean value of the parameter in windows of $1 \mathrm{keV}$ width below $20 \mathrm{keV}$ (around channel 300) is selected as corresponding to scintillation events. The efficiency of this cut is checked at low energy with the $3.2 \mathrm{keV}$ events. When this P1 cut is applied to the background, threshold is lowered considerably (see Figure 3) but not enough. New pulse parameters are being studied to develop multiparametric noise cuts.
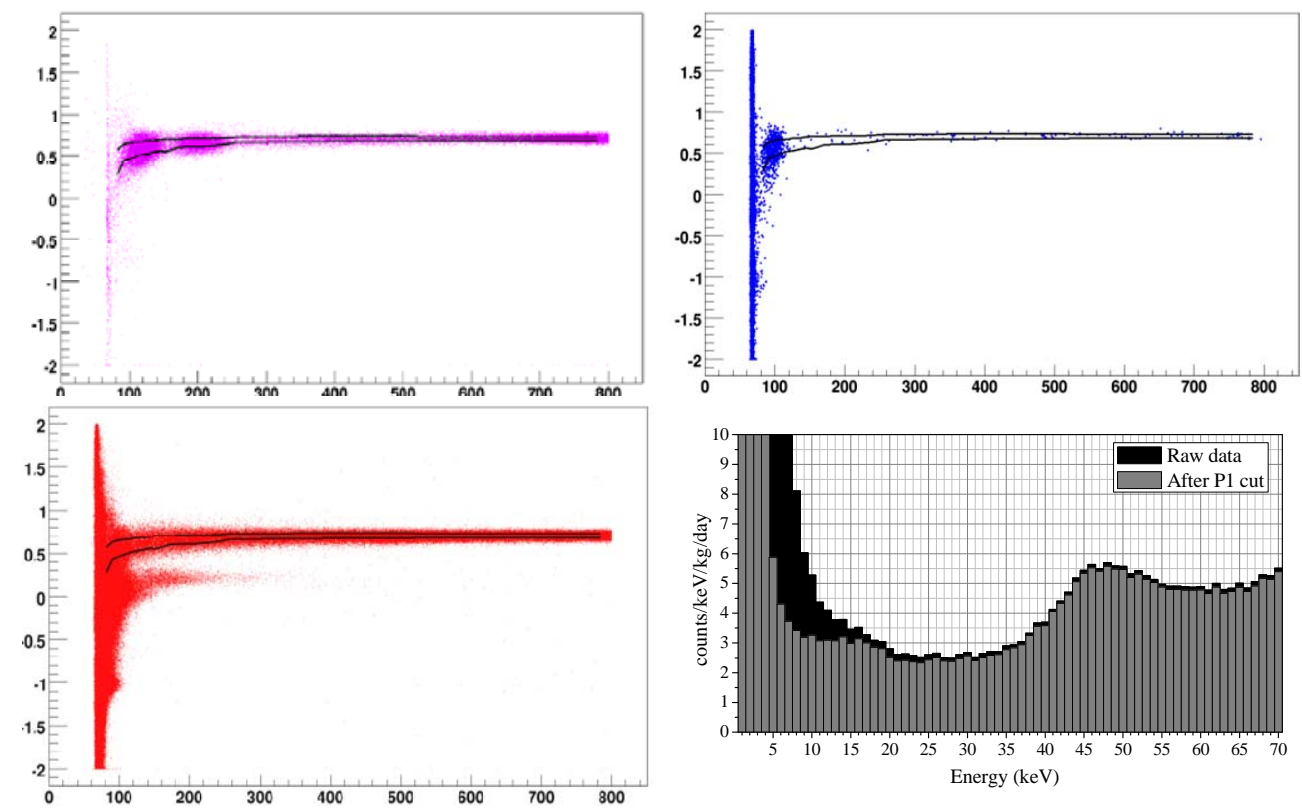

Figure 3: Parameter P1 vs. QDC for ${ }^{57} \mathrm{Co}$ (purple), ${ }^{40} \mathrm{~K}$ (blue) and background (red) events. Solid lines represent $1 \sigma$ region centred at the mean value of the parameter on the ${ }^{57} \mathrm{Co}$ calibration data, which is the cut applied to select scintillations events rejecting PMT noise.

\section{Light collection}

Different photomultipliers have been tested: from Electron Tubes Limited model 9302Blow-background (ETL) with Nominal Quantum Efficiency (NQE) 30\% and from Hamamatsu model R6233-100 one of High Quantum Efficiency (Ham HQE) with NQE $\geq 40 \%$ and other of low background (Ham LB) with NQE $\geq 35 \%$, also some preliminary tests with the Hamamatsu R11065SEL ultra low background (Ham ULB) with NQE $\geq 32 \%$. Tests were carried out in Zaragoza with a small commercial $\mathrm{NaI}(\mathrm{Tl})$ crystal, at Canfranc Underground Laboratory with the ANAIS-0 module and with a HP Ge detector for radiopurity testing. Tests showed that Hamamatsu photomultipliers have better resolution than the Electron Tubes one, Ham LB 
present better resolution than Ham HQE and light guides (LG) of $10 \mathrm{~cm}$ worsen energy resolution approximately a 20\% (see Figure 4).

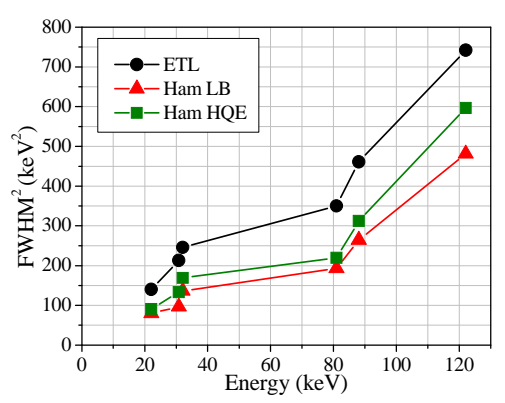

(a)

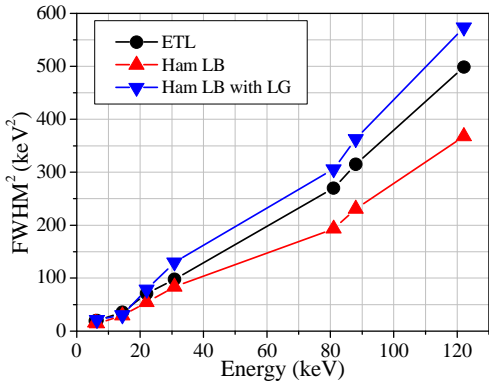

(b)

Figure 4: Resolution tests carried out with low energy calibration sources (a) Tests performed with a test bench in Zaragoza showed that Hamamatsu PMTs have better resolution than the one of Electron Tubes and that the resolution of Ham LB is slightly better than the one of Ham HQE. (b) Tests carried out underground with ANAIS-0 confirmed this and also showed that with light guides the resolution worsens approximately a $20 \%$.

For the light collection efficiency test, the parameter n (number of peaks in the pulse) is used since it could be an estimator of the number of detected photoelectrons. In the coincidence set-up, the calculation of $\mathrm{n}$ for $3.2 \mathrm{keV}\left({ }^{40} \mathrm{~K}\right)$ events, selected in $1 \sigma$ window of the corresponding QDC signal peak, gives as a preliminary result 7.5 photoelectrons $/ \mathrm{keV}$ (without light guides) (see Figure 5). In order to further study the effect of light guides in the light collection, the number of peaks in the pulse can be compared with and without light guides. This measurement was not carried out in the coincidence set-up and the $3.2 \mathrm{keV}$ events are not available. For this reason, low energy events from an external ${ }^{57}$ Co source are studied, although affected by surface effects which prevent from assigning them precisely the energy. Results obtained show that with light guides, light losses amount 36\%. However, light guides improve background at low and high energy to such an extent that with light guides, background is dominated by the crystal contamination.

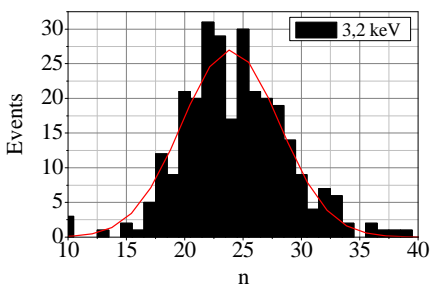

(a)

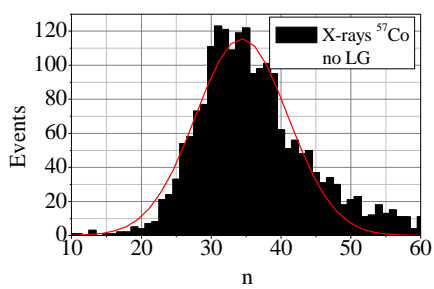

(b)

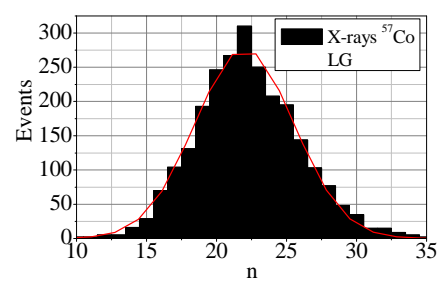

(c)

Figure 5: Calculation of the number of peaks in the pulse: (a) for $3.2 \mathrm{keV}$ events, gives a mean value $\mathrm{n}=23.95$. (b) For ${ }^{57}$ Co X-rays without light guides $\mathrm{n}=34.37$ and (c) with light guides $\mathrm{n}=21.99$. 
Radiopurity level of all the photomultipliers available has been measured at Canfranc Underground Laboratory (see Table 1). Ham ULB PMT has been only screened for radioactive contaminants and no direct information about resolution or efficiency is available because it was a special loan of the company for low background screening.

\begin{tabular}{ccccc}
\hline & \multicolumn{4}{c}{$\mathrm{mBq} / \mathrm{PMT}$} \\
\cline { 2 - 5 } Electron Tubes & $420 \pm 50$ & $24 \pm 4$ & $220 \pm 12$ & - \\
& ${ }^{40} \mathrm{~K}$ & ${ }^{232} \mathrm{Th}$ & ${ }^{60} \mathrm{Co}$ \\
\hline Ham HQE & $(185 \pm 1) \cdot 10^{3}$ & $420 \pm 40$ & $510 \pm 30$ & - \\
Ham LB & $678 \pm 42$ & $68 \pm 3$ & $100 \pm 3$ & - \\
Ham ULB & $32 \pm 9$ & $1.9 \pm 0.7$ & ${ }^{238} \mathrm{U}-33 \pm 7$ & $3.7 \pm 0.5$ \\
\hline
\end{tabular}

Table 1. Radiopurity measurements of all different photomultipliers at a HP Ge test bench in the Canfranc Underground Laboratory.

The sharing of the energy between the two PMTs in ANAIS-0 is observed to be asymmetric when plotting the signal of one PMT versus the other (see Figure 6a). According to calibrations, lateral bands correspond to background events coming from the sides (see Figure $6 b)$. Asymmetric events, not a huge amount ( $10 \%$ with light guides and $\sim 15 \%$ without light guides), also suggest contribution to the background from the photomultipliers. It is worth noting that asymmetric events present normal scintillation shape and must not be rejected. However, strongly asymmetric events, like those shown in black in Figure 6c, do not have the appropriate $\mathrm{NaI}(\mathrm{Tl})$ scintillation constant and can be rejected because they are non-bulk scintillation.

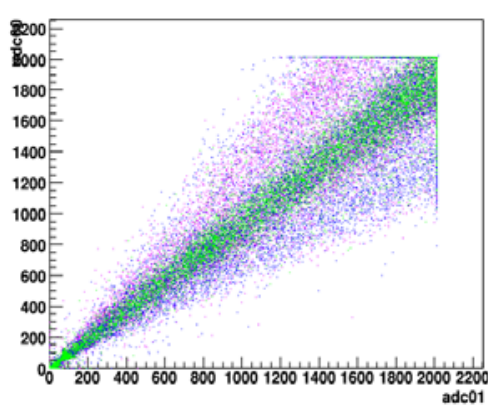

(a)

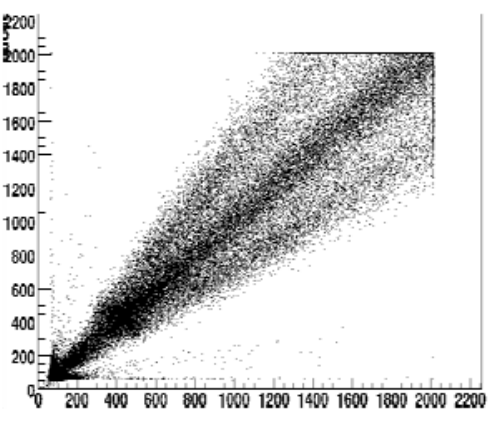

(b)

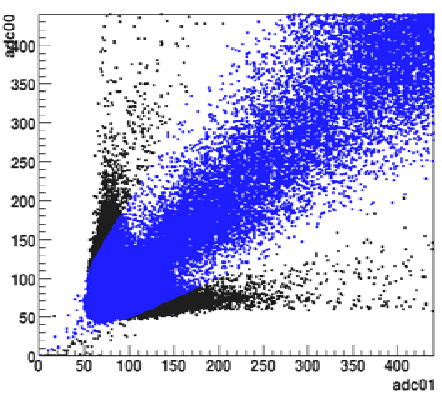

(c)

Figure 6: Asymmetry in the sharing of the energy between the two PMTs. (a) Results of low energy calibrations carried out at different locations on the crystal: at one end (purple) at the other end (blue) and at the centre (green). (b) Background measurement. (c) Background measurement enlarged. It presents strongly asymmetric events (black) that are non-bulk scintillation and can be distinguished from bulk scintillation ones (blue). 


\section{Conclusions}

A contract with Electrochemical Systems Inc. to purify NaI powder has been signed. If purification method is successful, $100 \mathrm{~kg}-250 \mathrm{~kg}$ will be purified along 2011.

There are on-going measurements in Canfranc Underground Laboratory with ANAIS-0 to characterize and fully understand ANAIS background at low energy, optimize noise cuts, determine the energy calibration method, test the electronics and improve the light collection efficiency.

Populations of scintillation events (from calibration sources and internal ${ }^{40} \mathrm{~K}$ ) at very low energy are analyzed. They are very useful to calibrate and reject noise in the region where the dark matter signal is searched for.

Light guides worsen considerably the collection of the light but they are necessary to reduce the contribution of the photomultipliers to the background. Ultra low background photomultipliers from Hamamatsu (R11065) could minimize this problem.

\section{Acknowledgments}

This work has been supported by the Spanish Ministerio de Ciencia e Innovación (MICINN) under project reference FPA2008-03228 and Consolider-Ingenio 2010 Programme under grant Multidark CSD2009-00064. C. Cuesta is supported by a Gobierno de Aragón predoctoral grant.

\section{References}

[1] R. Bernabei et al. (DAMA/LIBRA Collaboration), Eur. Phys. J. C 56 (2008) 333-355 (arXiv:0804.2741).

[2] Z. Ahmed et al. (CDMS Collaboration), Science 327 (2010) 1619.

[3] C. E. Aalseth et al. (CoGeNT Collaboration), Phys. Rev. Lett. in press, arXiv:1002.4703 [astroph.CO].

[4] J. Amaré et al., in Proceedings of the International Workshop on the Identification of Dark Matter (IDM 06). Rhodes, September 2006 (M. Axenides, G. Fanourakis and J.Vergados. Published by World Scientific, 2007).

[5] C. Pobes et al., in Proceedings of the International Workshop on the Identification of Dark Matter (IDM 06). Rhodes, September 2006 (M. Axenides, G. Fanourakis and J.Vergados. Published by World Scientific, 2007). 